\title{
A Low-Cost, Non-hazardous Protocol for Surface Texturing of Glass Particles
}

\author{
Anelechi Ibekwe $^{1} \cdot$ Yukie Tanino $^{1}$ (D) $\cdot$ Dubravka Pokrajac $^{1}$
}

Received: 5 August 2019 / Accepted: 19 September 2019 / Published online: 30 September 2019

(c) The Author(s) 2019

\begin{abstract}
We present a cheap, efficient, and non-hazardous protocol for altering the roughness of hard particles at the nanometer-scale using a stone tumbler, a tool which is normally used for polishing stones. Six different textures were achieved by lining the tumbler with sandpaper of mean grit diameters $d_{\mathrm{g}}=201,58.5,18.3,12.6$, and $8.4 \mu \mathrm{m}$. Two textures were created by tumbling a batch of glass spheres for $4 \mathrm{~h}$ and for $12 \mathrm{~h}$ with the $12.6 \mu \mathrm{m}$ sandpaper; all other textures were established by tumbling for $12 \mathrm{~h}$. Surface roughness was characterized by the integral length scale, $\xi$, evaluated from $7 \mathrm{~nm} /$ pix resolution scanning electron microscope images. Roughness size increased from $\xi=24$ to $31 \mathrm{~nm}$ as the grit size decreased from $d_{\mathrm{g}}=201$ to $18.3 \mu \mathrm{m}$, and then decreased to $\xi=6.4 \mathrm{~nm}$ at the smallest $d_{\mathrm{g}}$. The largest $\xi(=34 \mathrm{~nm})$ was achieved using a $12.6 \mu \mathrm{m}$ sandpaper and the shorter tumbling time of $4 \mathrm{~h}$. The permeability of a packed column of the particles broadly decreased with increasing $\xi$, indicating that permeability decreases with increasing roughness size.
\end{abstract}

Keywords Surface roughness $\cdot$ SEM $\cdot$ Abrasive wear $\cdot$ Glass $\cdot$ Polishing $\cdot$ Permeability $\cdot$ Surface metrology $\cdot$ Correlation length

\section{Introduction}

Surface roughness is important in many fields of engineering and natural sciences. It is well established that roughness influences the wetting nature of materials and surfaces [e.g., 1-5]. Processes such as ink-jet printing [6], painting, coating, biofouling, drying, lubrication, and waterproofing [7-9] all exemplify wetting principles. Similarly, previous studies of flow in porous media have reported correlations between grain roughness and capillary trapping [10, 11], capillary pressure-saturation curves [11], fracture conductivity $[12,13]$ and, most recently, pore-scale distribution in contact angle [14].

Surface texturing methods for flat materials (metals/ alloys, polymers, and glass) have evolved rapidly over the years. Techniques such as abrasive [15], chemical [16], and laser etching [17], as well as abrasive polishing [18, 19], blasting [20], mechanical press [21], electrical discharge machining [22], electron beam lithography [23],

Yukie Tanino

ytanino@abdn.ac.uk

1 School of Engineering, University of Aberdeen, Aberdeen, Scotland AB24 3UE, UK photolithography [24], and plasma nano-texturing [25] have all been applied to produce either random or structured rough surfaces. In contrast, established techniques for roughening batches of mm-scale particles is limited. In particular, the most widely used technique for roughening glass particles is chemical etching with hydrofluoric acid [e.g., 11, $26,27]$, which is highly corrosive and particularly hazardous. This paper presents a new method for surface texturing of glass particles that is safe, effective, and cheap. Treated particles are visualized using scanning electron microscopy (SEM), and their surface topography is characterized by its two-point spatial correlation function. The permeability of packed columns of particles was measured and its dependence on surface roughness discussed.

\section{Materials and Methods}

\subsection{Particle Texturing}

Soda-lime glass spheres (Mo-Sci Corporation, USA) of mean diameter $966 \pm 34 \mu \mathrm{m}$ (standard dev., 35 samples) 
were used in this study. The particles were textured using an off-the-shelf stone tumbler (UKGE, UK; Fig. 1a), a piece of household equipment commonly used to polish rock or

Table 1 Mean permeability and median integral length scale for each treatment condition

\begin{tabular}{lllll}
\hline $\begin{array}{l}\text { Treatment } \\
\text { condition }\end{array}$ & $d_{\mathrm{g}}(\mu \mathrm{m})$ & $\begin{array}{l}\text { Tumbling } \\
\text { time }(\mathrm{h})\end{array}$ & $k\left(\mu \mathrm{m}^{2}\right)$ & $\xi(\mathrm{nm})$ \\
\hline R0 & - & - & $460 \pm 27$ & $14.6 \pm 6.3$ \\
R1 & 201 & 12 & $438 \pm 19$ & $24.3 \pm 1.7$ \\
R2 & 58.5 & 12 & $407 \pm 12$ & $29.0 \pm 2.1$ \\
R3 & 18.3 & 12 & $399 \pm 14$ & $31.4 \pm 2.4$ \\
R4 & 12.6 & 4 & $364 \pm 10$ & $33.5 \pm 1.8$ \\
R5 & 12.6 & 12 & $499 \pm 14$ & $12.8 \pm 8.0$ \\
R6 & 8.40 & 12 & $493 \pm 14$ & $6.4 \pm 1.2$ \\
\hline
\end{tabular}

$\xi$ is the median over ten measurements for R0, nine measurements for $\mathrm{R} 1$, and eight measurements each for R2-R6; $k$ is the average of ten measurements for all treatment conditions. The uncertainty reported for $k$ and $\xi$ are standard deviations and standard errors of the mean, respectively stones. Here, we used the equipment to either smoothen or roughen glass surfaces by tuning the grit size of the sandpaper (Klingspor Abrasives, Germany) that lines the tumbler and the duration of treatment. In total, five grit sizes, $d_{\mathrm{g}}=201,58.5,18.3,12.6$, and $8.4 \mu \mathrm{m}$, where $d_{\mathrm{g}}$ is the average grit diameter, were considered. The tumbling duration was kept constant at $12 \mathrm{~h}$, except for one texturing regime comprising $d_{\mathrm{g}}=12.6 \mu \mathrm{m}$ sandpaper and a shorter tumbling time of $4 \mathrm{~h}$ (Table 1).

For each $d_{\mathrm{g}}$, approximately $20 \mathrm{~cm}^{3}$ of untreated particles were placed in a $97 \mathrm{~mm}$-long, $103 \mathrm{~mm}$-diameter barrel at a time and tumbled at $66 \mathrm{rpm}$ for the selected tumbling duration. After tumbling, the samples were first soaked in a $2 \%$ aqueous solution of Decon 90 for 2 to $24 \mathrm{~h}$ to remove the dust generated by the abrasive wear process. Subsequently, the samples were gently agitated by swirling, rinsed with warm water, and oven-dried at $100{ }^{\circ} \mathrm{C}$. Additionally, the samples were cleaned sequentially with toluene, propanol, and water, and then oven-dried again prior to surface roughness characterization.

\section{(a)}

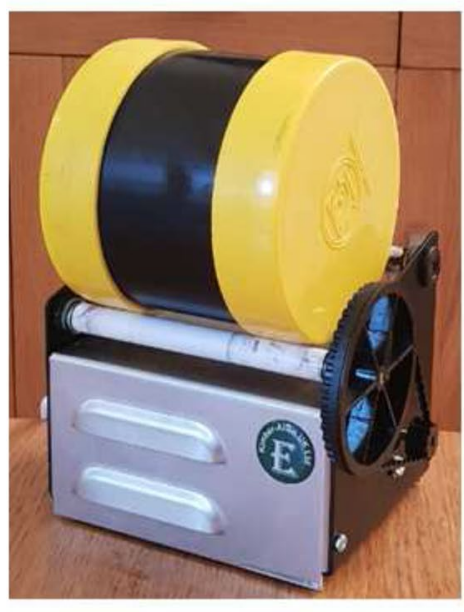

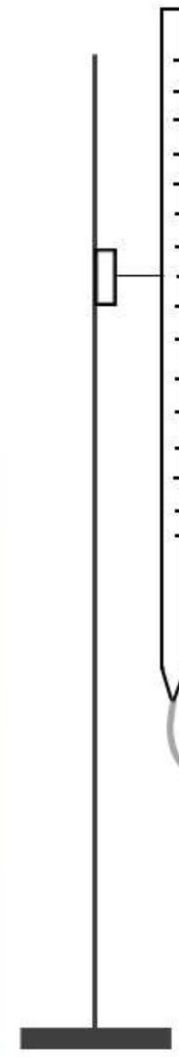

(b)

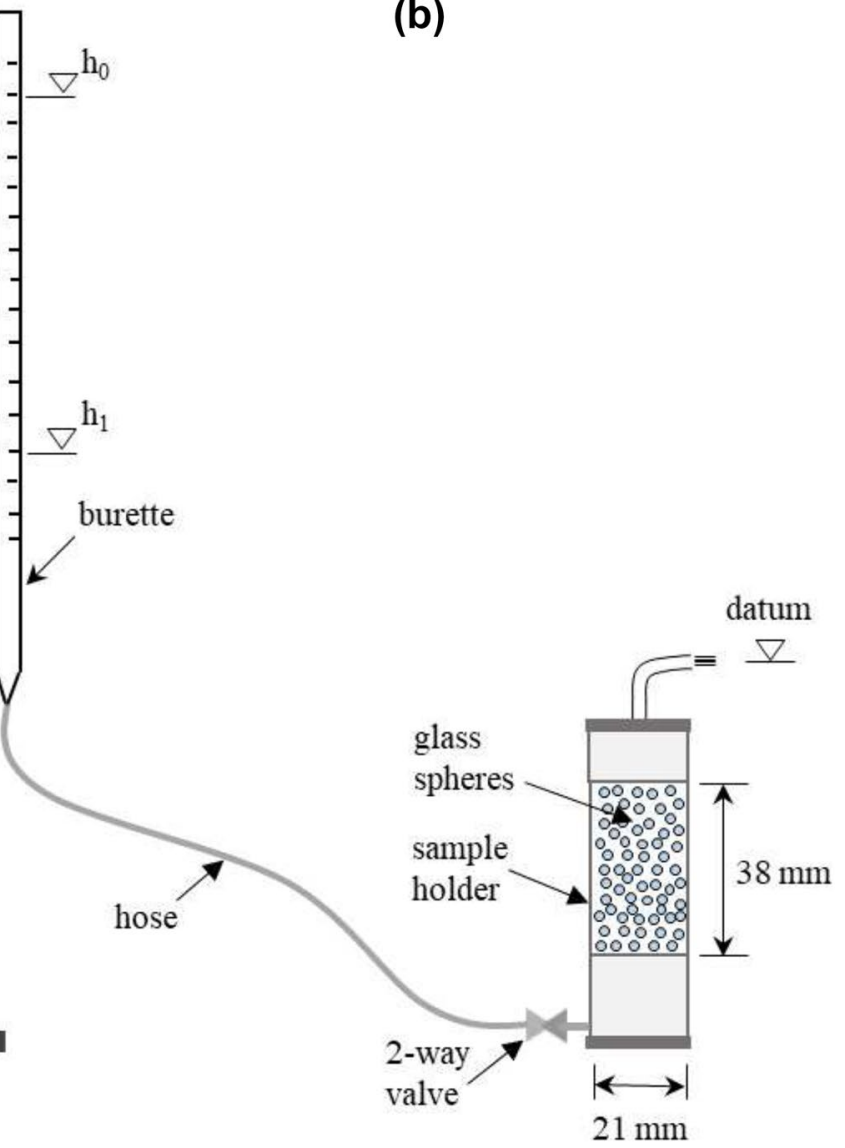

Fig. 1 a Stone tumbler. The plastic barrel (black) is $103 \mathrm{~mm}$ in diameter and $97 \mathrm{~mm}$ in length. During operation, the barrel is sealed with the yellow lids and rotates at $66 \mathrm{rpm}$. b Experimental setup for per-

meability measurement using the falling-head method. $h_{0}(=580 \mathrm{~mm})$ and $h_{1}(=80 \mathrm{~mm})$ represent the initial and final water levels, respectively (Color figure online) 


\subsubsection{Correlation Length}

To characterize the surface features, $8-10$ particles of each treatment condition were imaged at $16,000 \times$ magnification (corresponding image resolution $6.9 \mathrm{~nm} / \mathrm{pix}$ ) using a Carl Zeiss GeminiSEM 300 (e.g., Fig. 2). The two-point autocorrelation function, $Z(m, n)$, where $(m, n)$ are the lags in pixels in the two orthogonal directions, was computed for each image using the Wiener-Khintchine theorem [e.g., 28] and MATLAB's fft2.m and ifft2.m functions [29]. The tumbling was isotropic and, accordingly, $Z(m, n)$ was averaged at fixed radii, $r\left(=\sqrt{m^{2}+n^{2}}\right)$, following the approach of Berryman [30] to calculate the 1D, isotropic autocorrelation function, $\hat{Z}(r)$. Finally, the integral length scale, $\xi$, was evaluated as $\xi=\int_{0}^{r_{0}} \hat{Z}(r) \mathrm{d} r$, where $r=r_{0}$ is the first zero-crossing. The computed values of $\xi$ are summarised in Table 1; all $\hat{Z}(r)$ are presented in Fig. 3.

\subsection{Permeability}

Falling-head permeability tests were performed to further explore the effect of the proposed treatment. Glass spheres were wet-packed into a vertical column (ID $21 \mathrm{~mm}$, Fig. 1b). X-ray micro-computed tomography images of R0, R3, and R4 samples, acquired at voxel resolutions of $10 \mu \mathrm{m}$, yielded consistent porosity estimates of $35.6 \pm 0.2 \%$ (standard dev., 6 packed columns), confirming packing reproducibility. The upstream end of the packed column was connected to a burette (ID $11 \mathrm{~mm}$ ) through a hose (ID $4 \mathrm{~mm}$ ), while the outlet at the downstream end was open to the atmosphere. A test consisted of measuring time it takes for the water level in the burette to lower from the initial position, $h_{0}$, to another one, $h_{1}$, using a stopwatch. Both heights were measured from the datum located in the middle of the outlet. The experiment was repeated ten times to determine average values. The experiment was also carried out with the empty column to measure the flow resistance of the system without any particles. The falling head equation was modified by subtracting the resistance of the empty column in order to calculate corrected values of the absolute permeability.

\section{Results}

Figure 4 presents the integral length scale (Fig. 4a) and permeability (b) for each treatment condition. $\xi=14.3 \pm 6.3 \mathrm{~nm}$ (standard error) and $k=460 \pm 27 \mu \mathrm{m}^{2}$ (standard dev.) for untreated samples. This value of $\xi$ is one order of magnitude smaller than the vertical roughness length scale of untreated soda-lime glass spheres from the same supplier, but two orders of magnitude smaller than the lateral length scale [27], suggesting that $\xi$ as measured presently is a proxy for the size of features rather than their lateral spacing. The smallest $k\left(=360 \pm 10 \mu \mathrm{m}^{2}\right)$ coincides with the largest $\xi(=34 \mathrm{~nm})$, and was achieved using $d_{\mathrm{g}}=12.6 \mu \mathrm{m}$ sandpaper and the shorter tumbling time of $4 \mathrm{~h}$ (treatment R4, Table 1).

Particles tumbled for $12 \mathrm{~h}$ display two regimes. As the grit size decreased from $d_{\mathrm{g}}=201$ (R1) to $18.3 \mu \mathrm{m}$ (R3), the roughness size increased from $\xi=24.3 \pm 1.7$ to $31.4 \pm 2.4 \mathrm{~nm}$ and permeability decreased from $k=440$ to $400 \mu \mathrm{m}^{2}$. As the grit size decreased further, however, $\xi$ decreased with $d_{\mathrm{g}}$ until $\xi=6.4 \pm 1.2 \mathrm{~nm}$ at the finest grit size (R6), while permeability increased to $k=500 \mu \mathrm{m}^{2}$ at $d_{\mathrm{g}}=12.6 \mu \mathrm{m}(\mathrm{R} 5)$ beyond which it remained constant. This
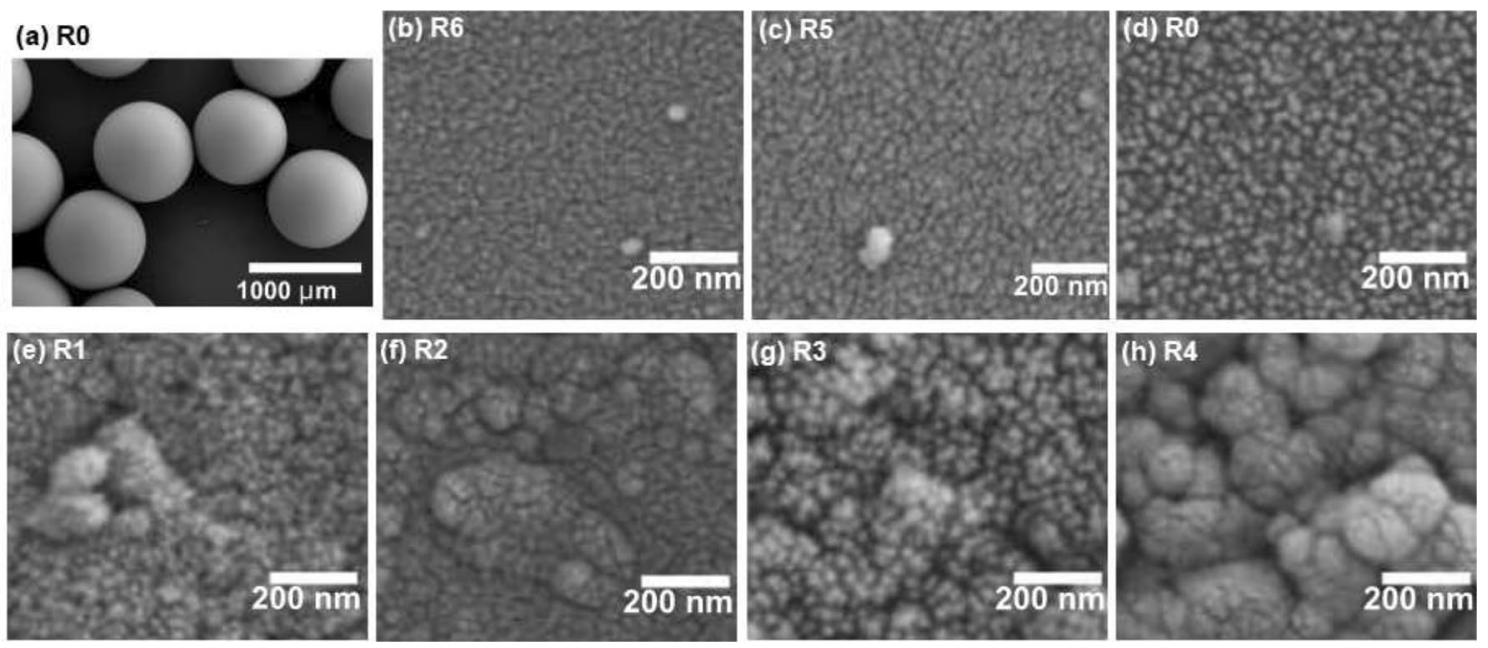

Fig. 2 Selected SEM images of R0 (a, d), R6 (b), R5 (c), R1 (e), R2 (f), R3 (g), and R4 (h) samples. The autocorrelation functions for images (b-h) are included in Fig. 3; the corresponding $\xi=6.6(\mathbf{b}), 7.9(\mathbf{c}), 14.7(\mathbf{d}), 24.3(\mathbf{e}), 29.3(\mathbf{f}), 33.5(\mathbf{g})$, and $33.9(\mathbf{h}) \mathrm{nm}$, respectively 
Fig. 3 Autocorrelation functions for ten samples of R0, nine samples of R1, and eight samples of R2-R6. Axis limits are identical in each plot; the abscissa axes are truncated at 50 pixels to reveal finer details of the plots
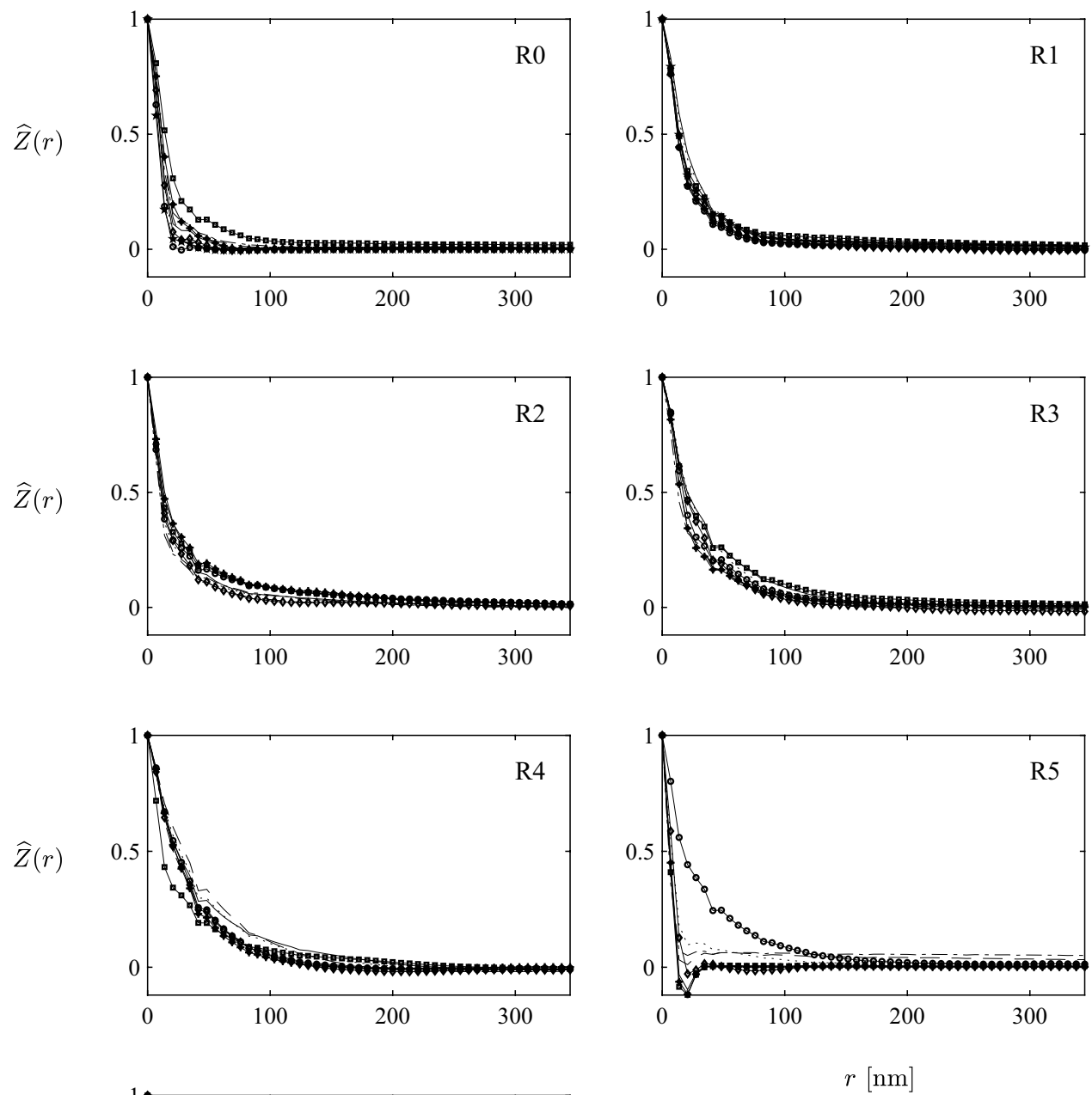

asymptotic limit is consistent with $k \sim 670 \mu \mathrm{m}^{2}$ predicted by Ergun [31]'s equation for packed columns of spheres.

Combined, $k$ decreased with increasing integral length scale (Fig. 4c). We infer from the negative correlation that, under conditions considered presently, permeability decreases with increasing roughness even under laminar conditions and $\xi$ is a measure of the vertical dimension of $\mathrm{nm}$-scale surface features. Plausible explanations for the negative correlation include the formation of recirculation zones within troughs and an increase in the particle surface area per particle with increasing $\xi$. Further insight requires comprehensive 3D topography measurements using sophisticated surface metrology techniques.

Treatment conditions R5 and R6 established $\xi$ smaller than those of untreated particles, indicating that, for a given tumbling time, fine grit smoothens the surface while coarse grit roughens it. The critical grit size between the two regimes, $d_{\mathrm{g}} \approx 15 \mu \mathrm{m}$, corresponds to $1.5 \%$ of the particle diameter. However, the critical grit size-and the properties of the treated surface in general - are expected to be a function of the hardness of the particle relative to the grit, the concentration of particles in the tumbler, tumbling speed, 

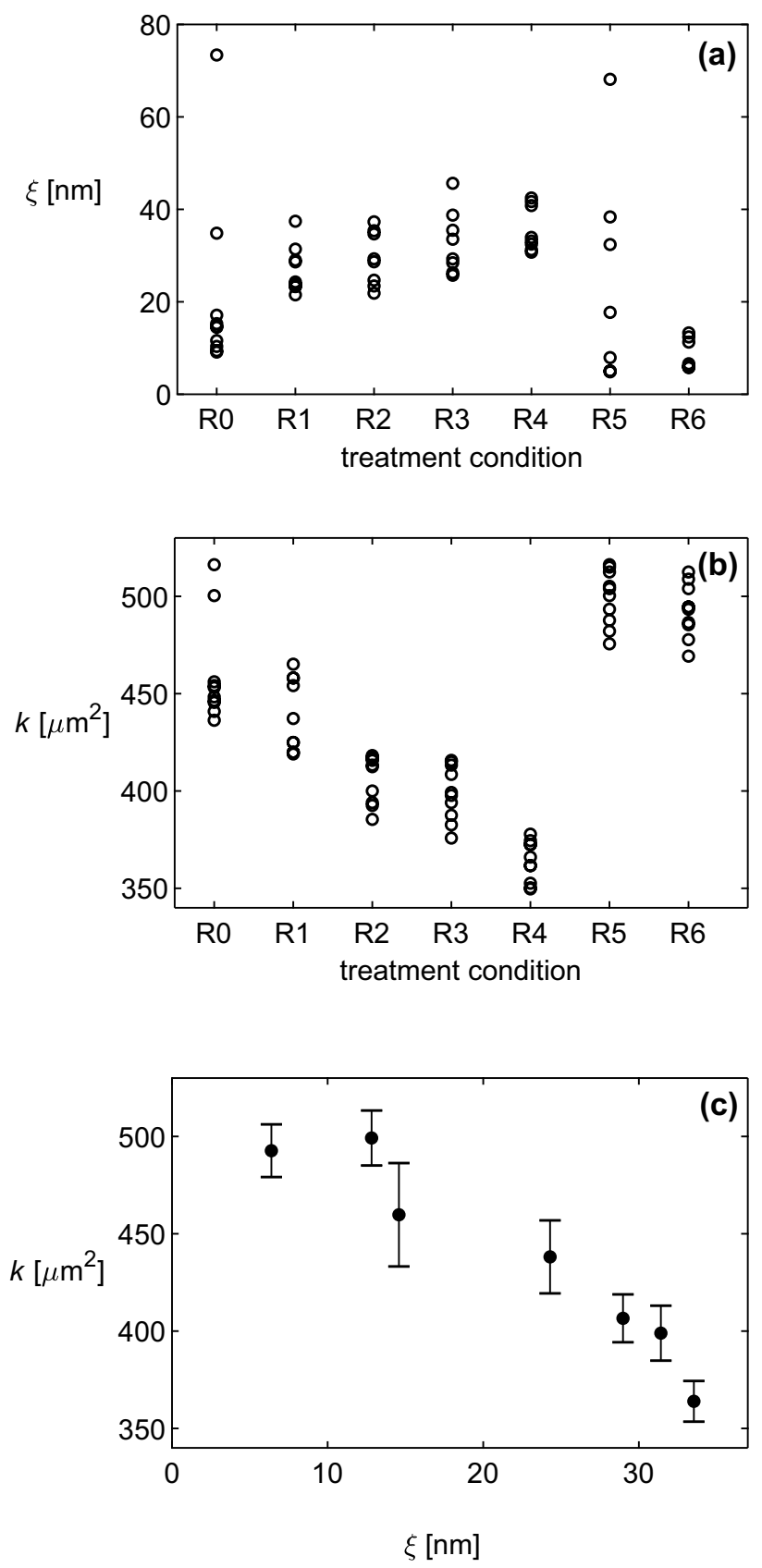

Fig. 4 The integral length scale (a) and permeability (b) at each treatment condition and the correlation between the two (c). Each open circle represents a different particle (a, b); solid circles represent the mean $k$ and median $\xi$ for each treatment condition. Vertical bars in (c) depict the standard deviations across ten replicate measurements in the same packed column. The values of $(\xi, k)$ are summarized in Table 1

and particle diameter in addition to the parameters varied in the present study, namely the grit size and tumbling time. A comprehensive exploration of the impact of these parameters on texturing remains a topic for future work.

\section{Conclusions}

A new protocol for nm-scale surface texturing of glass particles using a sandpaper-lined tumbler was presented. The method is cheap, effective, can be readily applied to particles of other rigid materials such as plastic and metal, and safer than the standard approach of acid etching applied to glass particles. Depending on the sandpaper grit size, tumbling was found to create or remove nm-scale roughness. The integral length scale of the radial autocorrelation function computed from $7 \mathrm{~nm} /$ pix resolution SEM images of the particles is negatively correlated with permeability, indicating that roughness affects permeability even under laminar conditions and that the integral length scale is an effective proxy for roughness size.

Packed beds of glass and plastic spheres are widely used as laboratory models for porous media [e.g., 11, 27, 32, 33]. The protocol presented here facilitates laboratory investigation of the impact of grain roughness on single phase and multiphase flow in porous media, a research area that has yet to be fully explored.

Acknowledgements This material contains work supported by an Aberdeen Formation Evaluation Society student bursary and a Society of Petrophysicists and Well Log Analysts Foundation grant. AI was supported by a University of Aberdeen School of Engineering Elphinstone Ph.D. studentship. Electron microscopy was performed in the ACEMAC Facility at University of Aberdeen; the authors thank John Still for the acquisition of the SEM images. The authors gratefully acknowledge Alfred R. Akisanya and Mark Gourlay for helpful suggestions on the texturing protocol. The authors thank the anonymous reviewer for his/her comments. All data used in this study are available from the corresponding author on reasonable request.

\section{Compliance with Ethical Standards}

Conflict of interest The authors declare that there is no conflict of interest.

Open Access This article is distributed under the terms of the Creative Commons Attribution 4.0 International License (http://creativeco mmons.org/licenses/by/4.0/), which permits unrestricted use, distribution, and reproduction in any medium, provided you give appropriate credit to the original author(s) and the source, provide a link to the Creative Commons license, and indicate if changes were made.

\section{References}

1. Drelich, J., Miller, J.D., Good, R.J.: The effect of drop (bubble) size on advancing and receding contact angles for heterogeneous and rough solid surfaces as observed with sessile-drop and captive-bubble techniques. J. Colloid Interface Sci. 179(1), 37-50 (1996)

2. Morrow, N.R.: The effects of surface roughness on contact angle with special reference to petroleum recovery. J. Can. Pet. Technol. 14(4), 42-53 (1975). https://doi.org/10.2118/75-04-04 
3. Cassie, A.B.D., Baxter, S.: Wettability of porous surfaces. T. Faraday Soc. 40, 546-551 (1944). https://doi.org/10.1039/TF944 4000546

4. Wenzel, N.R.: Resistance of solid surfaces to wetting by water. Ind. Eng. Chem. 28(8), 988-994 (1936)

5. Wang, J., Do-Quang, M., Cannon, J.J., Yue, F., Suzuki, Y., Amberg, G., Shiomi, J.: Surface structure determines dynamic wetting. Sci. Rep. 5, 8474 (2015). https://doi.org/10.1038/srep0 8474

6. Xu, R., Fleming, P.D., Pekarovicova, A.: The effect of ink jet paper roughness on print gloss. J. Imaging Sci. Technol. 49(6), 660-666 (2005)

7. Bhushan, B., Jung, Y.C.: Wetting study of patterned surfaces for superhydrophobicity. Ultramicroscopy 107(10-11), 1033-1041 (2007)

8. Bora, C.K., Flater, E.E., Street, M.D., Redmond, J.M., Starr, M.J., Carpick, R.W., Plesha, M.E.: Multiscale roughness and modeling of MEMS interfaces. Tribol. Lett. 19(1), 37-48 (2005)

9. Ritcher, K., Feist, W.C., Knaebe, M.T.: The effect of surface roughness on the performance of finishes. Part 1. Roughness characterization and stain performance. For. Prod. J. 45(7-8), 91-97 (1995)

10. Geistlinger, H., Ataei-Dadavi, I., Mohammadian, S., Vogel, H.J.: The impact of pore structure and surface roughness on capillary trapping for 2-D and 3-D porous media: comparison with percolation theory. Water Resour. Res. 51(11), 9094-9111 (2015)

11. Dullien, F.A.L., Zarcone, C., Macdonald, I.F., Collins, A., Bochard, R.D.E.: The effects of surface roughness on the capillary pressure curves and the heights of capillary rise in glass bead packs. J. Colloid Interface Sci. 127(2), 362-372 (1989). https:// doi.org/10.1016/0021-9797(89)90042-8

12. Develi, K., Babadagli, T.: Experimental and visual analysis of single-phase flow through rough fracture replicas. Int. J. Rock Mech. Min. Sci. 73, 139-155 (2015)

13. Crandall, D., Bromhal, G., Karpyn, Z.T.: Numerical simulations examining the relationship between wall-roughness and fluid flow in rock fractures. Int. J. Rock Mech. Min. Sci. 47(5), 784-796 (2010)

14. AlRatrout, A., Blunt, M.J., Bijeljic, B.: Wettability in complex porous materials, the mixed-wet state, and its relationship to surface roughness. Proc. Nat. Acad. Sci. USA 115(36), 8901-8906 (2018). https://doi.org/10.1073/pnas.1803734115

15. Meiron, T.S., Marmur, A., Saguy, I.S.: Contact angle measurement on rough surfaces. J. Colloid Interface Sci. 274(2), 637-644 (2004)

16. Qian, B., Shen, Z.: Fabrication of superhydrophobic surfaces by dislocation-selective chemical etching on aluminum, copper, and zinc substrates. Langmuir 21(20), 9007-9009 (2005). https://doi. org/10.1021/la051308c

17. Calignano, F., Manfredi, D., Ambrosio, E.P., Iuliano, L., Fino, P.: Influence of process parameters on surface roughness of aluminum parts produced by DMLS. Int. J. Adv. Manuf. Tech. 67(9-12), 2743-2751 (2013)

18. Kubiak, K.J., Wilson, M.C.T., Mathia, T.G., Carval, P.: Wettability versus roughness of engineering surfaces. Wear 271(3-4), 523-528 (2011). https://doi.org/10.1016/j.wear.2010.03.029

19. de Moura, L.F., Hernández, R.E.: Effects of abrasive mineral, grit size and feed speed on the quality of sanded surfaces of sugar maple wood. Wood Sci. Technol. 40(6), 517-530 (2006). https:// doi.org/10.1007/s00226-006-0070-0
20. Rosales-Leal, J.I., Rodriguez-Valverde, M.A., Mazzaglia, G., Ramon-Torregrosa, P.J., Diaz-Rodriguez, L., Garcia-Martinez, O., Vallecillo-Capilla, M., Ruiz, C., Cabrerizo-Vilchez, M.A.: Effect of roughness, wettability and morphology of engineered titanium surfaces on osteoblast-like cell adhesion. Colloid Surf. A 365(1-3), 222-229 (2010)

21. Karim, A.M., Rothstein, J.P., Kavehpour, H.P.: Experimental study of dynamic contact angles on rough hydrophobic surfaces. J. Colloid Interface Sci. 513(1), 658-665 (2018)

22. Giljean, S., Bigerell, M., Anselme, K., Haidara, H.: New insights on contact angle/roughness dependence on high surface energy materials. Appl. Surf. Sci. 257(22), 9631-9638 (2011)

23. Jeong, H.E., Kwak, M.K., Park, C.I., Suh, K.Y.: Wettability of nanoengineered dual-roughness surfaces fabricated by UV-assisted capillary force lithography. J. Colloid Interface Sci. 339(1), 202-207 (2009). https://doi.org/10.1016/j. jcis.2009.07.020

24. Geistlinger, H., Ataei-Dadavi, I., Vogel, H.J.: Impact of surface roughness on capillary trapping using $2 \mathrm{D}$-micromodel visualization experiments. Transp. Porous Med. 112(1), 207-227 (2016). https://doi.org/10.1007/s11242-016-0641-y

25. Fischer, G., Drahi, E., Foldyna, M., Germer, T.A., Johnson, E.V.: Plasma nanotexturing of silicon surfaces for photovoltaics applications: influence of initial surface finish on the evolution of topographical and optical properties. Opt. Express 25(24), A1057A1071 (2017). https://doi.org/10.1364/OE.25.0A1057

26. Hassas, B.V., Caliskan, H., Guven, O., Karakas, F., Cinar, M., Celik, M.: Effect of roughness and shape factor on floation characteristics of glass beads. Colloid Surf. A 492(5), 88-99 (2016). https://doi.org/10.1016/j.colsurfa.2015.12.025

27. Utermann, S., Aurin, P., Benderoth, M., Fischer, C., Schroter, M. Tailoring the frictional properties of granular media. Phys. Rev. E 84, 031306 (2011). https://doi.org/10.1103/PhysRevE.84.031306

28. Robertson, C., George, S.C.M.D.: Theory and practical recommendations for autocorrelation-based image correlation spectroscopy. J. Biomed. Opt. 17(8), 080801 (2012). https://doi. org/10.1117/1.JBO.17.8.080801

29. Ursell, T.: autocorr2d. MATLAB Central File Exchange (2018). Accessed 12 June 2019

30. Berryman, J.G.: Measurement of spatial correlation functions using image processing techniques. J. Appl. Phys. 57, 2374-2384 (1985)

31. Ergun, S.: Fluid flow through packed columns. Chem. Eng. Prog. 48(2), 89-94 (1952)

32. Bowden, S.A., Tanino, Y., Akamairo, B., Christensen, M.: Recreating mineralogical petrographic heterogeneity within microfluidic chips: assembly, examples, and applications. Lab Chip 16, 4677-4681 (2016). https://doi.org/10.1039/C6LC01209D

33. Shellenberger, K., Logan, B.E.: Effect of molecular scale roughness of glass beads on colloidal and bacterial deposition. Environ. Sci. Technol. 36(2), 184-189 (2002). https://doi.org/10.1021/ es015515k

Publisher's Note Springer Nature remains neutral with regard to jurisdictional claims in published maps and institutional affiliations. 